\title{
$\nabla$ Aquasomes: A Self-Assembled Nano-Particulate Carrier System
}

\section{IJCRR}

Section: Healthcare

ISI Impact Factor

(2019-20): 1.628

IC Value (2019): 90.81

$\operatorname{SJIF}(2020)=7.893$

\section{Ajay Kumar Gupta, Diptee Gupta, Vedant Gupta}

University lnstitute of Pharmacy, Chhatrapati Shahu Ji Maharaj University, Kanpur - 208024, Uttar Pradesh, India

\section{ABSTRACT}

In the last few decades, nanobiotechnology emerged as a novel approach for those drugs that face challenges to deliver in conventional dosage forms. Nanoparticles, liposomes, niosomes, quantum dots and aquasomes are some major different type of nano-biotechnologically developed carrier system. The aquasomesare one of the emerging approach and ideal choice of drug delivery comprises of the nano-particulate self-assembled carrier system. In the development of ceramic nanoparticles, aquasomes proved as a significant drug delivery system. Aquasomes are the three-layered structure, fabricated from the solid crystalline core, coated with carbohydrates on to which biologically active drug molecules are adsorbed. The solid core confers the structural stability, whereas the polyhydroxy oligomer coating protects against dehydration and confers stability to active drug molecules. Formulations of aquasomes are mainly administered by parenteral route but new studies suggest that it could also be administered by other routes. Aquasomes delivers their bioactive molecules via a combination of special targeting molecular shielding and sustained release process. Hydroxyapatite core-based aquasomes are broadly used for the preparation of implants. Aquasomes possess properties of maintaining conformational integrity, and a high degree of surface exposure, which is successfully targeted for the delivery of peptide molecules such as insulin, haemoglobin; enzymes like serratiopeptidase and also aid in targeting vaccine and gene to specific sites. The present article is an attempt to focus on the possible revolutionary applications of aquasomes.

Key Words: Nanobiotechnology, Aquasomes, Carrier system, Novel drug delivery

\section{INTRODUCTION}

Aquasomes are nano-particulate carrier system but instead of being simple nanoparticles, these are three-layered self-assembled structures, comprised of a solid phase nano-crystalline core coated with an oligomeric film to which biochemically active molecules are adsorbed with or without modification. ${ }^{1}$ So "aquasomes" are carbohydrate stabilized nanoparticles of the core which was first developed by NirKossovsky in 1995. Alternatively, aquasomes are termed as "Bodies of Water", their water like properties support and sustain fragile biological molecules such as polypeptide and proteins. The function of preserving confirmative integrity and a high degree of surface exposure is used to target and deliver bioactive molecules such as peptides and protein hormones, antigens and genes to specific locations where the action is required. ${ }^{2}$

The aquasomes made up of ceramic core are stabilized by carbohydrates and by using methods like co-polymerization, diffusion or adsorption; the pharmacologically active molecules are incorporated on to the carbohydrate surfaces of preformed nanoparticles. ${ }^{3}$ Aquasomes composed of threelayered structure: solid crystalline core, carbohydrate coat and the active drug which are self-assembled by non-covalent bonds (Figure 1). The core is coated with polyhydroxy oligomers onto which bioactive molecules are adsorbed. The solid core provides structural ability while carbohydrate coating plays an important role and acts like a natural stabilizer which protects against dehydration and stabilizes the biochemically active molecules. ${ }^{4}$

Aquasomes are spherical 60-300 nm particles. Mainly three types of core materials are used for developing aquasomes i.e. Tin oxide, Nanocrystalline carbon ceramics (diamonds) and Brushite (calcium phosphate dihydrate). Aquasomes offer an attractive mode of delivery for drugs which are having problems such as route of delivery, physical as well as chemical instability, poor bioavailability and potent side effects. ${ }^{5}$

\section{Corresponding Author:}

Diptee Gupta, Research Scholar, Department of Pharmaceutics, University Institute of Pharmacy, Chhatrapati Shahuji Maharaj University, Kanpur - 208024, Uttar Pradesh, India; Email: gdiptee98@gmail.com

ISSN: 2231-2196 (Print)

Received: 10.08 .2020
ISSN: 0975-5241 (Online)

Revised: 05.10 .2020
Accepted: 12.11 .2020
Published: 16.02 .2021 
The discovery of aquasomes include concept from food chemistry, microbiology, biophysics and frequent discoveries including solid-phase synthesis, supramolecular chemistry, molecular shape change and self-assembly. ${ }^{6}$

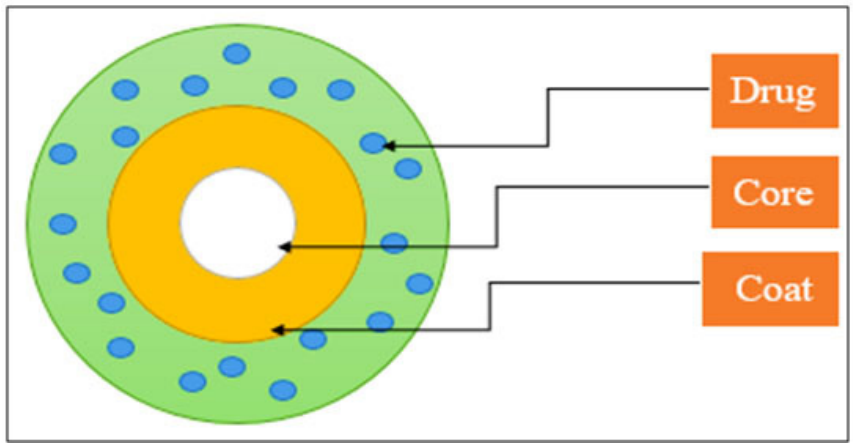

Figure 1: Schematic diagram of Aquasome. It has 3 layers. 1. Solid Crystalline Core 2. Polyhydroxy oligomer coat 3. Bioactive molecules.

\section{PROPERTIES OF AQUASOMES}

- Aquasomes prevent clearance via the reticuloendothelial system or degradation by other environmental problems because of their size and structural stability.

- Due to its large size and active surface, Aquasomes can be loaded with adequate amounts of agents via non-covalent, ionic bonds, van der Waals and entropic forces successfully.

- The mechanism of action of aquasomes is governed by their surface chemistry.

- The combination of various processes like specific targeting, molecular shielding, and slow and sustained release processes involved in the delivery of the active drug via aquasomes. ${ }^{8}$

- Water like properties of aquasomes provides a platform for preserving the conformational integrity and biochemical stability of bio-active.?

- Aquasomes as a carrier also protects the drug/antigen/ protein from harsh $\mathrm{pH}$ conditions and enzymatic degradation, thus requiring lower doses. ${ }^{6}$

\section{PRINCIPLE OF SELF-ASSEMBLY}

Self-assembly means that in two or three-dimensional space, the constituent parts of any final product assume the spontaneously prescribed structural orientations. The three-layered structure are self-assembled by non-covalent bonds. ${ }^{7}$ Self-assembly in the aqueous environment of macromolecules have the virtue to design itself in smart nanostructured materials, which is primarily governed by three physicochemical processes;

a. Interaction between charged groups: Interactions of charged groups such as amino, carboxyl, sulphate, and phosphate-groups, facilitate the long-range approach of self-assembling subunits. From the biological environment, the intrinsic chemical groups or adsorbed ions impart a polarity charge to biological and synthetic surfaces. Most biochemically related molecules are amphoteric molecules. ${ }^{9}$ For the first phase of self-assembly, the long-range interaction of constituent subunits begins at an intermolecular distance of around $15 \mathrm{~nm}$. Long-range forces can extend to $25 \mathrm{~nm}$ in the case of hydrophobic structures. Charged groups also play a part in the stabilisation of folded protein tertiary structures.

b. Hydrogen bonding and dehydration effect: Hydrogen bond plays an important role in base pair matching and help to stabilize the secondary protein structure such as alpha helices and beta sheets. Hydrophilic molecules that form hydrogen bonds, provides the surrounding water molecules with a significant degree of organisation. Whereas hydrophobic molecules unable to form hydrogen bonds, having the ability to repel water molecules from their surroundings to organize the moiety. Organized water decreases the entropy of the surrounding environment. Since it is thermodynamically unfavourable, the molecule dehydrates and get self-assembled. ${ }^{10}$

c. Structural stability: In the biological environment, structural stability of protein determined by the interaction between charged group and hydrogen bonds largely external to the molecule and by Van der Waals forces which responsible for hardness and softness of molecule and maintenance of internal secondary structures, provides sufficient softness, allows maintenance of conformation during self-assembly. ${ }^{9}$ Van der Waals forces often experienced by the relatively hydrophobic molecular regions that are shielded from water, play a subtle but critical role in maintaining molecular conformation during self-assembly. ${ }^{11}$

In the case of aquasomes, sugars help in molecular plasticization. ${ }^{12}$ Van der Waals forces also play a small but measurable role in the interaction of polypeptides with carbohydrates and related polyhydroxy-oligomers. When molecules change their shape considerably following an interaction, the energy minima assumed upon conformational denaturation tend to block reversal. ${ }^{10}$

\section{OBJECTIVES OF AQUASOMES}

- The main objective of preparing aquasomes is to protect bio-actives.

- Aquasomes maintain molecular conformation and optimum pharmacological activity. ${ }^{9}$

- Many other delivery systems like pro-drugs, liposomes are prone to destructive interactions between drug and carrier while aquasomes have carbohydrate coating prevents destructive denaturing interaction between drug and solid carriers. ${ }^{13}$ 
- Aquasomes with natural stabilizers like various polyhydroxy sugars act as dehydroprotectant, help in maintaining water-like state and preserves molecules in dry solid-state, protecting from the change in aqueous state, $\mathrm{pH}$, temperature, solvent, salt causing denaturation. ${ }^{8,14}$

- An active molecule has characteristics such as unique three-dimensional conformation, freedom of internal molecular rearrangement caused by the freedom of bulk motion molecular interactions, but protein initiates irreversible denaturation when desiccated, even unstable in an aqueous state. ${ }^{10,14}$

\section{STRATEGIES USED IN THE CHEMICAL SYN- THESIS OF AQUASOMES}

Aquasomes are self-assembled three-layered nanostructure molecules. Hence the strategies involved in chemical synthesis of these nanostructures discussed below.

a. Sequential covalent synthesis: This strategy is used to produce arrays of covalently linked atoms with well-defined composition, connectivity and shape such as Vitamin $B_{12}$, it can produce the structures that are distant from the thermodynamic minimum for collection of atoms. ${ }^{15}$

Covalent polymerization: This strategy is used to prepare molecules with high molecular weight. Low weight substances are permitted to react with itself to yield molecules, including many covalently associated monomers. ${ }^{15}$ Such as Formation of polyethene from ethylene. Covalent polymerization indirectly provides synthetic routes to stable nanostructures and phase-separated polymers. ${ }^{3}$

Self-organizing synthesis: This strategy depends on weaker and less directional bonds such as hydrogen, ionic and Van der Waals interactions to assemble atoms, ions or molecules into structures. ${ }^{8}$ The different types of formulation are prepared by the use of this strategy include molecular crystals, ligand crystals, emulsions, colloids, micelles, self-assembled monolayers and phase-separated polymers. The ion or molecules adjust their position to reach the thermodynamic minimum and get self-organize, during formulation. ${ }^{16}$

Molecular self-assembly: Self-assembly is a process in which a disorganized structure with pre-existing components forms an organized structure or design. Aquasomes self-assembly has various interesting applications in nanoscience and nanotechnology formulation development. ${ }^{15,16}$

\section{COMPOSITION OF AQUASOMES}

a. Core material: Widely used core materials are ceramic and polymers. Ceramics such as diamond particles, brushite (calcium phosphate) and tin oxide are crys- talline easy to manufacture, biodegradable in nature, low cost and biocompatible. It provides a high degree of order and structural regularity. Due to the high degree of order, higher surface energy yields which leads to efficacious binding of carbohydrate onto it. These properties making it a good candidate for aquasome formulation. ${ }^{17}$ Polymers such as albumin, gelatin or acrylate are used. ${ }^{18}$

b. Coating material: Cellobiose, pyridoxal 5 phosphate, sucrose, trehalose, chitosan, citrate etc. are widely used coating materials. As a natural stabilizer, carbohydrate plays a crucial role and it is preferred mostly. Carbohydrates are adsorbed as a glassy film in nanometre size range coating the preformed ceramicnanoparticles and self-assembled calcium phosphate dihydrate particles (colloidal precipitation). ${ }^{19}$ The carbohydrate used this purpose are as

c. Cellobiose: It is 4-O-beta-D-glucopyranosyl-D-glucopyranose reducing sugar. It is acquired from the partial hydrolysis of cellulose. It protects the drug molecule against the dehydration. ${ }^{20}$

d. Trehalose: It is an alpha-D-glucopuyranosyl-alphaD-glucopyranoside non-reducing sugar. Trehalose also shields the drug molecule against dehydration and denaturation. It is observed more effective than cellobiose. ${ }^{20}$ It shown to be tolerant of stress in fungi, bacteria, insects, yeast and some plants. Trehalose acts by protecting proteins and membranes within plant cell during the desiccation process and thus preserves cell structures, inherent flavours, colours and texture. ${ }^{21}$ Disaccharides like sucrose, trehalose contain a large number of hydroxyl groups and help to replace the water with polar protein residues. Thus it maintains their integrity in the absence of water. Experimental studies done with Calcium transporting microsomes isolated from rabbit muscles and lobster muscles showed that the structure and function of cellular components could be protected by sugar during lyophilisation. ${ }^{22}$ The rehydrated vesicles displays drastically decreased calcium-uptake and uncoupled activity of ATPase when Calcium transporting microsomes are lyophilized without stabilizer sugar. Lyophilized vesicles are morphologically distinct from newly prepared vesicles in presence of as little as $0.3 \mathrm{~g}$ of trehalose per g. membrane upon rehydration..$^{21,23}$

e. Bio-active molecules: Drugs which have the property of interacting with the film via non-covalent and ionic interactions proved a good candidate for aquasomes. ${ }^{22}$

Among three layers of aquasomes, carbohydrate fulfils the objective of aquasomes. The hydroxyl groups on carbohydrate interact with polar and charged groups of proteins, in the same way as with water thus preserve the aqueous structure of proteins on dehydration. ${ }^{23}$ 


\section{METHOD OF PREPARATION OF AQUASOMES}

Aquasomes preparation is a very simple and effortless process which require minimum solvent usage and no homogenization steps. By using the principle of self-assembly, the aquasomes are prepared in three steps, i.e., Formation of the core, coating of the core, and immobilization of drug molecule (Figure 2). ${ }^{4}$

Formation of core material: The first step in the formulation of aquasomes is the development of the ceramic core. The method of ceramic core preparation depends on the choice of core materials. These ceramic cores can be built by various processes such as colloidal precipitation, sonication, inverted magnetron sputtering, and plasma condensation etc. ${ }^{24}$ In the preparation of core, the most regular material preferred which is ceramic. Two ceramic cores that are generally used diamond and calcium phosphate.

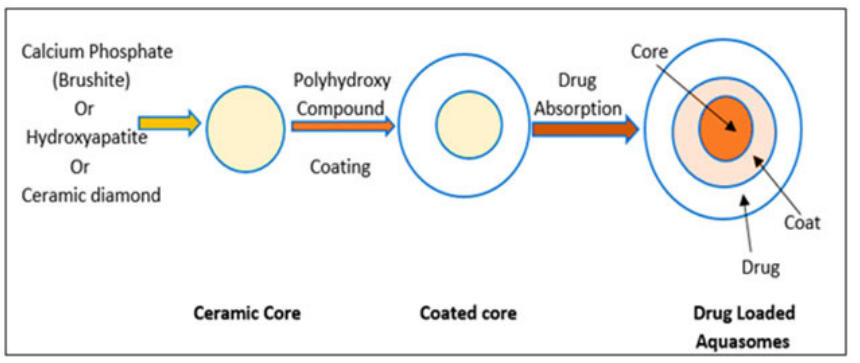

Figure 2: Method of preparation of aquasomes. It involves three steps- a) Ceramic core is prepared first by a different process such as colloidal precipitation, sonication etc. b) $\ln 2^{\text {nd }}$ step ceramic core is coated by polyhydroxy compound c) In $3^{\text {rd }}$ step loading of the drug is done by partial adsorption.

\section{a) Synthesis of Nanocrystalline tin oxide core}

It can be synthesized by direct current reactive magnetron sputtering. Under a high-pressure mixture of argon and oxygen, the high purity tin is blown from a diameter of 3 inches. Ultrafine particles deposited on a copper tube in a gaseous phase and cooled to $77 \mathrm{~K}$ with the flow of nitrogen. ${ }^{3}$

\section{b) Self-assembled Nanocrystalline brushite (calcium phosphate dihydrate)}

It can be synthesized by various processes such as co-precipitation, self-precipitation, sonication and PAMAM methods.

(i) Co-precipitation: Diammonium hydrogen phosphate solution is added dropwise to calcium nitrate solution with continuous stirring. The temperature is maintained at $75^{\circ} \mathrm{C}$ in a flask bearing a charge funnel, a thermometer, a reflux condenser fitted with a carbon dioxide trap. ${ }^{24,}{ }^{25} \mathrm{The} \mathrm{pH}$ of calcium nitrate is maintained 8-10 using the concentrated aqueous ammonia solution. Under the above-mentioned condition, the mixture is magnetically stirred. The precipitates are then filtered, washed and finally dried overnight. In an electric furnace, the powder was sintered by heating to $800-900^{\circ} \mathrm{C}^{26}$.

(ii) Sonication: Using ultrasonic bath, the solutions of disodium hydrogen phosphate and calcium chloride were mixed and sonicated. Equivalent moles of both reagents are used. For 2 hrs, the temperature was maintained at $4{ }^{\circ} \mathrm{C}$. The ceramic core is separated by centrifugation and then washed, resuspended in distilled water and filtered. The core material retained on filter paper is collected and dried appropriately.

(iii) Poly (Amidoamine) PAMAM: PAMAM was dissolved in the simulated body fluid of $\mathrm{pH} 7.4$ and placed it for 1 week at $37^{\circ} \mathrm{C}$ to induce nucleation and crystal growth. By the addition of the $\mathrm{NaOH}$ solution, the $\mathrm{pH}$ of the solution was adjusted. The precipitate formed was washed multiple times with deionized water. Then it was filtered and dried overnight. ${ }^{28}$

c) Nanocrystalline carbon ceramic, diamond particle: These ceramic may also be used for the core synthesis after ultra-cleansing and sonication. The main property of these core is crystalline. ${ }^{3}$

\section{d) Coating of the core with polyhydroxy oligomer}

Commonly used coating materials are cellobiose, citrate, pyridoxal 5 phosphate, trehalose and sucrose. It is the second step in which ceramic cores are coated with carbohydrates. By the addition of carbohydrate into an aqueous dispersion of the cores under sonication, the coating is carried out. These are then subjected to lyophilization which provides irreversible adsorption of carbohydrate onto the ceramic surface. The unadsorbed carbohydrate is removed by centrifugation. ${ }^{26}$

\section{e) Immobilization of drug molecule}

Loading of the drug to coated particles by partial adsorption is the final step for the preparation of aquasomes. A solution of known concentration of the drug is prepared at suitable $\mathrm{pH}$ buffer. Coated particles are dispersed and at low temperature, dispersion is kept at overnight for drug loading or lyophilized. ${ }^{27}$ After sometime drug-loaded formulation obtained, then characterized using various techniques.

\section{FATE OF AQUASOME}

Self-assembled aquasomes are biodegradable nanoparticles that accumulate more in liver and muscles. ${ }^{21}$ The drug's pharmacological or biological activity can be accomplished instantly as it is detected without any surface alteration on the surface of the system and cannot find any difficulty in identifying receptor on the active site. ${ }^{29}$

In vivo studies predict, biodegradation of ceramic is achieved by monocytes and multicellular cells called osteoclasts because they intervene first at the biomaterial implantation site 
during an inflammatory reaction. ${ }^{30}$ Two types of phagocytosis process were observed-when cells come in contact with biomaterial; either calcium phosphate crystals were taken up alone and then dissolved in the cytoplasm after the disappearance of the phagosome membrane or dissolution after the formation of the hetero phagosome. ${ }^{31}$ Phagocytosis of calcium phosphate coincided with autophagy and the deposition of residual bodies in the cell.

\section{EVALUATION PARAMETER OF AQUASOMES}

Aquasomes mainly evaluated and characterized by their various morphological and structural property of their core and polyhydroxy oligomer coating structure.

\section{a. Evaluation parameter for core material}

\section{Size distribution}

Scanning electron microscopy (SEM) and transmission electron microscopy (TEM) techniques are used for particle size distribution and morphological analysis. ${ }^{25}$ To determine the particle size, samples were placed on the surface of a specimen stub coated with gold using double-sided adhesive tape in SEM while in case of TEM, particle size is determined after negative staining with phosphotungstic acid. ${ }^{31}$ Coated core, as well as drug-loaded aquasomes are also analysed by these techniques.

\section{Characterization (FTIR)}

Fourier transform infrared spectroscopy (FT-IR) spectroscopy used for determining structural analysis. Potassium bromide sample disk method is used. ${ }^{32}$ Both the core and coated core can be analyzed by recording their IR spectra in the wavenumber range $4000-400 \mathrm{~cm}^{1}$. The characteristic peaks are observed and tally with reference peaks. ${ }^{33}$ Stability of the drug in the formulation can be also determined by this technique.

\section{X-ray diffraction}

To study crystalline or amorphous nature of a material X-ray diffraction study is performed. The hydroxyapatite ceramic core is analysed by exposing the core to copper $(\mathrm{Cu})$, potassium $(\mathrm{K})$ radiation in a wide-angle $\mathrm{X}$-ray diffractometer. ${ }^{30}$ After that the X-ray diffraction pattern of the sample is compared with the standard diffractogram, based on which the interpretations are made. ${ }^{32}$ In a study, it was observed that calcium phosphate core, lactose individually gave identical sharp peaks for crystalline peaks but when carbohydrate coated cores were observed, peaks represented an amorphous structure..$^{34}$ It may be the reason for the coating technique (solubilization of carbohydrate insolvent and subsequent drying by lyophilization) and saturation of the surface of the core with carbohydrate (Figure 3 ). ${ }^{35}$

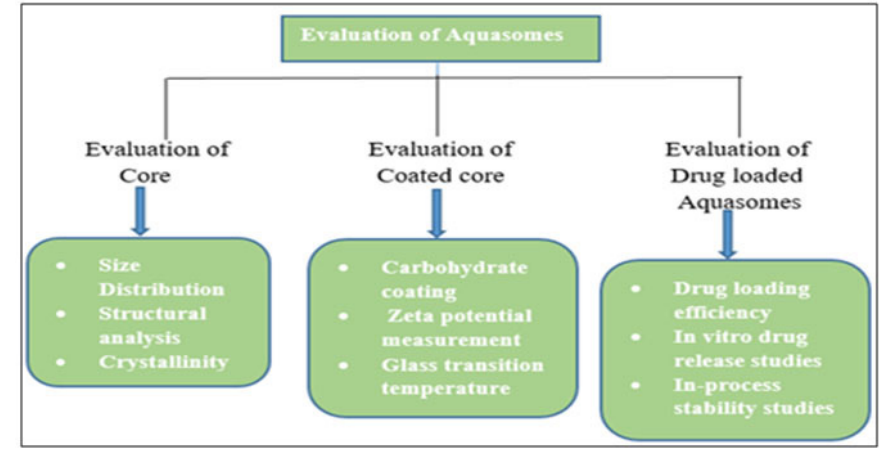

Figure 3: Evaluation parameter of aquasomes. Aquasomes consist of 3 layers and all 3 layers are evaluated for evaluation of aquasomes. In the figure, all methods for evaluation of each layer is enlisted.

\section{Evaluation parameter for coated core}

Carbohydrate coating: Coating of sugar over the ceramic core can be confirmed by Concanavalin A-induced aggregation method, Anthrone reaction and Phenol sulphuric acid method. In table 1 these methods are described.

\section{Zeta potential measurement}

The adsorption of sugar over the core and the prediction of storage stability determined by the measurement of zeta potential. Some studies indicated that with the increase in the saturation process by carbohydrate on to the hydroxyapatite core, the more decrease in zeta potential value. ${ }^{33}$

\section{Table 1: Method of Carbohydrate Coating ${ }^{31,36}$}

\section{S. Method Description No.}

1 Anthrone reaction

It is an example of the calorimetric method and used to quantify the unbound residual sugar or residual sugar remaining after coating. Anthrone reagent is added to sample and heated in a boiling water bath, cooled quickly. Under acidic conditions, carbohydrate gets hydrolysed to hydroxymethylfurfural which reacts with anthrone reagent to yield bluegreen colour complex. Absorbance is recorded $\left(\lambda_{\max }=625 \mathrm{~nm}\right)$ by using a UV-Visible spectrophotometer using glucose as standard 


\section{Table 1: (Continued)}

\begin{tabular}{lll}
$\begin{array}{l}\text { S. Method } \\
\text { No. }\end{array}$ & Description \\
\hline $\begin{array}{l}\text { Concanavalin-A in- } \\
\text { duced aggregation }\end{array}$ & $\begin{array}{l}\text { Used to quantify the amount } \\
\text { of sugar-loaded on the ceramic } \\
\text { core. Concanavalin-A solution is } \\
\text { added to suspensions of differ- } \\
\text { ent carbohydrate coated core (in } \\
\text { quartz cuvettes). Absorbance is } \\
\text { recorded in UV-Visible spectro- } \\
\text { photometer at a wavelength of } \\
\text { 45o nm as a function of time of } 5 \\
\text { min interval. The obtained data } \\
\text { is subtracted from the blank } \\
\text { experiment } \\
\text { It is also a colourimetric method } \\
\text { used to find out total carbohy- } \\
\text { drate such as mono-, di-, oligo-, } \\
\text { acid method } \\
\text { and polysaccharides present } \\
\text { in the sample. Carbohydrate is } \\
\text { dehydrated to furfural deriva- } \\
\text { tive in presence of concentrated } \\
\text { sulphuric acid which is further } \\
\text { reacted with phenol to produce } \\
\text { yellow gold colour }\end{array}$ \\
&
\end{tabular}

\section{Glass transition temperature}

Differential Scanning Calorimetry (DSC) studied used to analyse the glass transition temperature of carbohydrates and protein. DSC used to study the effect of carbohydrate on the drug-loaded to aquasomes. ${ }^{34}$ The transition from glass to rubber state can be measured using a DSC analyser as a change in temperature upon melting of glass.

\section{b. Evaluation parameter of drug-loaded aquas- omes}

\section{Drug loading efficiency}

It is done to evaluate the amount of drug which is bound on the surface of aquasomes. The drug loading can be determined by incubating the aquasome formulation without the drug in a known concentration of the drug solution for $24 \mathrm{hrs}$ at $4{ }^{\circ} \mathrm{C} .{ }^{29}$ After that supernatant is separated by high-speed centrifugation for $1 \mathrm{hrs}$ at low temperature in a refrigerated centrifuge. ${ }^{37}$ Then the clear extractive supernatant is filtered and analyzed free drug content by UV spectrophotometer. The drug payload/drug loading is calculated by the following formula-

$\%$ Drug loading $=$ (Weight of total added drug-weight of the unentrapped drug $) /($ Weight of aquasomes $) \times 100$

\section{In vittro drug release studies}

The in vitro release kinetics of the loaded drug is done to study the release pattern of the drug from the aquasomes. Incubate a known quantity of drug-loaded aquasomes in a buffer of suitable $\mathrm{pH}$ at $37^{\circ} \mathrm{C}$ with continuous stirring..$^{35}$ Samples are extracted from time to time and centrifuged for some periods at high speeds. After each withdrawal, equivalent medium volumes must be substituted. Then supernatants are analyzed to estimate the amount of released drug. ${ }^{38}$

\section{c. In-process stability studies}

SDS-PAGE (sodium dodecyl sulphate polyacrylamide gel electrophoresis) can be used to assess the stability and integrity of protein during the formulation of the aquasomes. ${ }^{36}$

\section{ADVANTAGE OF AQUASOMES}

- Aquasomes systems act as a reservoir to release the molecules either in a continuous or a pulsatile manner, avoiding a multiple-injection schedule. ${ }^{39}$

- Aquasomes based vaccines offer many advantages as a vaccine delivery system. Cellular and humoral immune responses can be elicited to antigens adsorbed on to aquasomes. ${ }^{36}$

- Aquasomes improves the pharmaceutically active agent's therapeutic effectiveness and defends the medication from phagocytosis and degradation. ${ }^{36}$

- These nanoparticles offer a favourable environment for proteins thereby avoiding their denaturalization.

- Enzyme activity and molecular conformation sensitivity have made aquasome a novel carrier for enzymes such as DNaseand pigment/dyes.

- Multi-layered aquasomes conjugated with biorecognition molecules such as antibodies, nucleic acid, peptides which are known as biological labels can be used for various imaging tests. ${ }^{39}$

\section{APPLICATION OF AQUASOMES}

\section{a. Oral delivery of acid-labile enzyme}

Rawat et al developed a nanosized ceramic core-based system for oral administration of the acid-labile enzyme serratiopeptidase, prepared by colloidal precipitation under sonication at room temperature coated with chitosan under constant stirring. By further encapsulating the enzyme-loaded core into an alginate gel, the enzyme was secured. The TEM images of particles showed them with a spherical shape and an average diameter of $925 \mathrm{~nm} .{ }^{40}$ Particle enzyme-loading was found to be approximately $46 \%$. Both stability and integrity of enzyme during formulation steps was evaluated by in vitro proteolytic activity. The results revealed these aquasomes protect the structural integrity of enzymes, resulting in a more potent therapeutic effect. ${ }^{31}$

\section{b. Insulin and Insulinomimetics delivery}

The parenteral delivery of insulin, via aquasomes, done by Cherian et a.l using a calcium phosphate ceramic 
core. Several disaccharides such as trehalose, cellobiose, and pyridoxal-5-phosphate used for coating the core. The drug loading to the coated cores performed via adsorption process. ${ }^{32}$ The in vivo activity of aquasome formulations of insulin assessed by using albino rats. Pyridoxal-5-phosphate-coated particles found more effective in reducing blood glucose levels as compared to particles coated with Trehalose or Cellobiose. The prolonged activity as a result of the slow release of drug from the carrier as well as the structural stability of the peptide. ${ }^{41}$

Insulinomimetics aquasomes formulated by colloidal precipitation. Disodium hydrogen phosphate and calcium chloride solution sonicated at low temperature and then cores were coated with disaccharides and immediately loaded with polypeptide- $\mathrm{k}$. In vivo activity evaluated by using albino Wistar rats. ${ }^{42}$ Studies indicated that trehalose coated aquasomes released polypeptide- $\mathrm{k}$ faster than cellobiose coated aquasomes. It also reported that polypeptide- $\mathrm{k}$ oral delivery did not cause any significant change in serum glucose level. ${ }^{43}$

\section{c. Delivery of antigens}

The adjuvants usually used to boost the antigen immunity appear either to modify antigen confirmation by surface adsorption or to shield the functional groups. The effectiveness of a new adapted ceramic antigen delivery vehicle is thereby formulated and evaluated by Kossovskyet al. These particles were diamond substrate wrappedin an aqueous dispersion with a glassy carbohydrate (cellobiose) layer and immunologically active surface molecule. ${ }^{38}$ These aquasomes having size range 5-300nm provided both conformational stabilization and a high degree of surface exposure to protein antigen. ${ }^{42}$ Due to high surface energy, the diamond was the first choice for adsorption and adhesion of cellobiose. It provided a colloidal surface which is capable of hydrogen bonding to the proteinaceous antigen. ${ }^{40}$ The disaccharide helps to minimize the surface-induced denaturation of adsorbed antigens (muscle adhesive protein, MAP). ${ }^{42}$

In other studies, BSA (Bovine serum albumin) loaded aquasomes formulated by self-assembling of hydroxyapatite using the co-precipitation method. Trehalose and cellobiose have been used as coating materials. The antigen-loading efficiency was found to be about $20-30 \%$. This BSA loaded aquasomes showed more potent immunological activity compared to that of plain bovine serum albumin, after SC injection. ${ }^{21,36}$ Aquasomes of malarial merozoite surface protein 119 (MSP-119) were formulated by co-precipitation method using hydroxyapatite nano-ceramic carriers. The small size and large surface area of the prepared hydroxyapatite showed good absorption immunogens efficiency. Slower in vitro antigen release and slower biodegradability activity were also seen in prepared nanoceramic formulations, which may lead to prolonged exposure to antigen-presenting cells and lymphocytes..$^{40,43}$

\section{d. As oxygen transporter}

In one study, hydroxyapatite core was prepared by Khopade et a.l using carboxylic acid-terminated half-generation poly (amidoamine) dendrimers, covered with trehalose trailed by adsorption of haemoglobin..$^{44,45}$ The particle size, drug loading capacity and oxygen-binding properties of formulation were studied. In vivo studies carried out in rats demonstrated that aquasomes have good potential for use as an oxygen carrier and prove effective to retain its oxygen-binding characteristics over 30 days. $^{28}$

\section{e. Delivery of enzymes}

Aquasomes also used for delivery of enzymes like DNase and pigment/dyes because enzymes activity fluctuates with molecular conformation and cosmetic properties of pigment are sensitive to molecular conformation. ${ }^{40} \mathrm{DNase}$ a therapeutic enzyme used in the treatment of cystic fibrosis was successfully immobilized on aquasomes and targeted to the specific site and elicited significant therapeutic effect as desired. Marked retention of biological activity was observed with surface-immobilized DNase on the solid phase of a colloidal calcium phosphate nanoparticle coated with polyhydroxyl oligomeric films. ${ }^{46}$

\section{e. Delivery of gene}

For effective targeted intracellular gene therapy, aquasomes have been used. Studies indicated that aquasomes protect and maintain the structural integrity of the gene segment. A five-layered composition comprised of ceramic core, polyhydroxy oligomeric film, therapeutic gene segment, additional carbohydrate film ${ }^{47}$ and a targeting layer of conformationally conserved viral membrane protein as shown in figure 3.

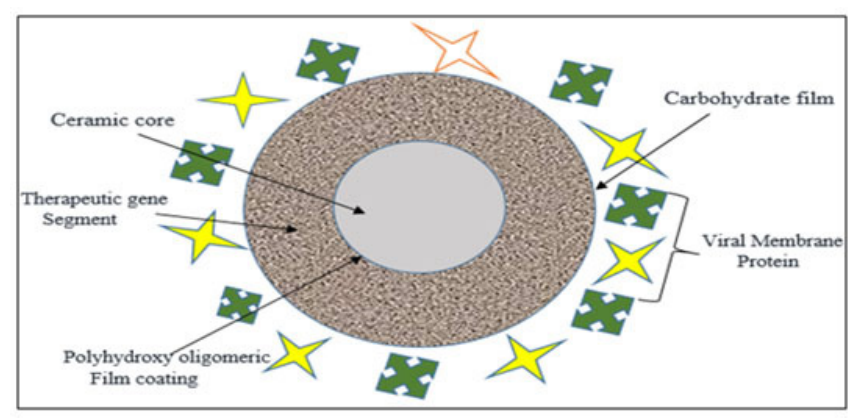

Figure 4: Gene delivery through aquasomes. Five layered compositions comprise the structure of aquasomes for gene delivery as 1 . Carbohydrate film 2. Ceramic core 3 . Therapeutic gene segment 4 . Polyhydroxy oligomeric coating 5 . Viral membrane protein.

\section{f. Delivery of Non-Protein Molecules}

Anti-cancer drug etoposide, when administered as aquasomes, is preferentially targeted to liver, spleen, lungs and kidney. A topical cream formulation of aqua some dithranol 
has been reported to lead to a more sustained release of the drug from its formulation. ${ }^{48}$

Table 2: Application of Aquasomes in the Delivery of Various Products

\begin{tabular}{|c|c|c|}
\hline Active Ingredient & Therapeutic Application & Reference \\
\hline Dithranol & Treatment of psoriasis & 45 \\
\hline Insulin & Glucose regulation & 20 \\
\hline Serratiopeptidase & $\begin{array}{l}\text { To improve proteolytic } \\
\text { activity }\end{array}$ & 44 \\
\hline Etoposide & Anticancertargeting & 46 \\
\hline $\operatorname{IFN} \alpha$ & Hairy cells & 47 \\
\hline Haemoglobin & $\begin{array}{l}\text { Blood component, the } \\
\text { oxygen carrier }\end{array}$ & 27 \\
\hline Hepatitis B vaccine & $\begin{array}{l}\text { Antigen for prevention of } \\
\text { jaundice }\end{array}$ & 32 \\
\hline Serum Albumin & $\begin{array}{l}\text { Maintain osmotic pressure, } \\
\text { needed for proper distribu- } \\
\text { tion of body fluids between } \\
\text { intravascular compart- } \\
\text { ments and body tissues }\end{array}$ & 49 \\
\hline $\begin{array}{l}\text { MSP } 119 \text { (Merozo- } \\
\text { ite surface protein) }\end{array}$ & Anti-malarial & 19 \\
\hline Indomethacin & $\begin{array}{l}\text { To increase the solubility as } \\
\text { well as to increase release } \\
\text { of drug }\end{array}$ & 35 \\
\hline
\end{tabular}

Application of aquasomes in the delivery of various products reported in Table 2. There are some active ingredients which therapeutic activity enhanced by formulating in aquasome novel drug delivery system.

\section{LIMITATIONS OF AQUASOMES}

There are some limitations which create a hindrance in formulating self-assembled aquasomes system. If the drug is poorly absorbed, it may cause burst release in the body that cause toxicity. To prevent opsonisation and phagocytic clearance of aquasomes in body, its surface could be coated with polyethene glycol. ${ }^{50}$

\section{FUTURE PERSPECTIVES}

Aquasome, a self-assembled system provide a promising future in the efficient delivery of the broad range of drug molecules including viral antigens, haemoglobin and insulin. The unique carbohydrate coating of the core show better biological activity and maintain the qualities of the drug molecule and its structural integrity. Biosensors are those devices that deliver the drug or those agents which monitor the drug and help in diagnosis. If biosensor is incorporated with the aqua some core, it may be effective to examine soft tissue in cancerous disease and also help in diagnosis. At a present time world is suffered from a pandemic covid-19 and there is no effective line of therapy to treat this. If the concept of slow antigen release in small quantity via aqua some which produce specific antibody in the body at the sustained rate is used in case of covid 19. It could be proven effective to enhance specific immunity against covid19. Along with immunity loss, it also has mild symptoms such as difficulty in breathing, decreased oxygen level that could also be maintained with the oxygen transport property of aquasomes.

\section{CONCLUSION}

Thus aquasomes based strategy provides pharmaceutical scientists with new hope for the delivery of a wide range of bioactive molecules and in the effective possible treatment of various diseases.

\section{ACKNOWLEDGEMENT}

The author wishes to express their sincere thanks to the Honourable Vice-chancellor of Chhatrapati Shahu Ji Maharaj University, Kanpur for motivating and inspiring to write this futuristic article.

Conflict of Interest: The author declares no conflict of interest.

\section{Source of Funding: Nil}

\section{REFERENCES}

1. Chandra D, Yadav KK, Singh VK, Patel A, Chaurasia S. An overview: The novel carrier for vesicular drug delivery system. World J Pharm Res 2014;3(6):1299-322.

2. Pandey S, Badola A, Bhatt GK, Kothiyal P. An overview on aquasomes. Int J Pharm Chem Sci 2013;2(3):1282-1287.

3. Jagdale S, Karekar S. Bird's eye view on aquasome: formulation and application. J Drug Deliv Sci Tech 2020;58:1-14.

4. Gulati M, Singh SK, Kaur P, Yadav AK, Pondman KM, Kishore U. Aquasomes: the journey so far and the road ahead. NanoNPP Mc Graw Hill, USA:2014:105-111.

5. Swain S, Beg S, M. Babu S. Liposheres as a novel carrier for lipid-based drug delivery: current and future directions. Rec Pat Drug Deliv Formul 2016;10(1):59-71.

6. Kumar J, Kumar VV, Mounica R, Bolla SP, Pavani M.Aquasomes-the best carriers for protein and peptide delivery. Asian J Pharm Res Dev 2013;1(4):16-23.

7. Parashar A. Literature review on aquasomes A drug carrier system. Indian J Med Res Pharma 2017 Nov; 4(11):27-30.

8. Jain S, Jain NK, Jain NK.Liposomes as drug carriers. In: Jain NK editors. Controlled and novel drug delivery,1st ed.New Delhi: CBSPublishers \& Distributors; 1997:304-352.

9. Wani SU, Yerawar AN. Aquasomes: a novel nanocarrier for drug delivery. IJPT 2010 Dec;2(4):446-457. 
10. Sharma S,Gupta A, Niranjan H, Goyal M.Aquasome: A Novel Drug Delivery Approach Using Nanocrystaline Biomaterial. Curr Res Pharm Sci 2012;01:16-21.

11. Shruti R, Alpana R, Shraddha P.Distinct Nanoparticles For Drug Delivery. Adv Res Pharm 2018; 8(4):1073-1081.

12. Jeong Y, Nah J W, Kwang H, Na K, Kim I, Cho C, Kim S. SelfAssembling Nanospheres ofHydrophobized Pullulans in Water. Drug Dev Ind Pharm 1999;25(8):917-927.

13. Chakraborty A, Lahkar S, Dey BK, Alam F. Aquasome - brief overview. Int J Pharm Sci Res 2014;2(6):1222-1230.

14. Rewar S. Avital role of Aquasome's on controlled and novel drug delivery. Int J Curr Res Chem Pharma Sci 2015;2(5):6-11.

15. Gholap AD, Bordue SS, Mahajan AM. Aquasomes: A potential drug delivery carrier. Pharmacology Online 2011:230-237.

16. Kajbafvala A, Bahmanpour H, Maneshian MH, Li M. Self-assembly techniques for nanofabrication. J Nanomater 2013:1-3.

17. Mandal S, Mandal M.Current Status and Future Prospects of New Drug Delivery System. Pharma Times 2010;42(4):13-17.

18. Hnatyszyn HJ, Kossovsky N, Gelman A, Sponsler E. Drug delivery systems for the future. Pharm Sci Technol 1994;48:247254.

19. Goyal AK, Khatri K, Mishra N, Mehta A, Vaidya B, Tiwari S. Development of self-assembled nanoceramic carrier construct(s) for vaccine delivery. J Biomater Appl 2009;24:65-84

20. Cherian AK, Rana AC, Jain SK. Self-assembled carbohydratestabilized ceramic nanoparticles for the parenteral delivery of insulin. Drug Dev Ind Pharm 2000;26(4):459-463.

21. Sahoo CK, Ramana DV, Satynarayan K, Mohanty D. Drug delivery through aquasomes. J Pharm Adv Res 2018;1(3):156-162.

22. Rege K, Huang HC, Barua S, Sharma G, DeySK. Inorganic nanoparticles for cancer imaging and therapy. J Cont Rel 2011;155(3):344-357.

23. Kaushik JK, Bhat R. Why is trehalose an exceptional protein stabilizer? An analysis of the thermal stability of proteins in the presence of the compatible osmolyte Trehalose. J Biol Chem 2003;278(29):26458-26465.

24. Virbhadra V, Jangme CM, Patil SS, Inde GS, Chavan DV, Yedale AD. A Review on aquasomes: a potential drug delivery carrier. Int Res J Pharm App Sci 2013;3(2):124-129.

25. Narang N. Aquasomes: self-assembled systemsfor the delivery of bioactive molecules. Asian J Pharm 2012;6:95-100

26. Vengala P, Dintakurthi S, Subrahmanyam CVS. Lactose coated ceramic nanoparticles for oral drug delivery. J Pharm Res 2013;7:540-545.

27. Khopade AJ, Khopade S, Jain NK. Development of haemoglobin aquasomes from sphericalhydroxyapatite cores precipitated in the presence ofhalf-generation poly(amidoamine) dendrimer. Int J Pharm 2002;241:145-154.

28. Kossovsky N, Gelman A, Sponsler E.Cross Linking Encapsulated Hemoglobin with Solid Phase Supports: Lipid Enveloped Hemoglobin Adsorbed to Surface Modified Ceramic Particles Exhibit Physiological Oxygen Lability. Artif Cells Nanomed Biotechnol 1994;22(3):479-485.

29. Mesariya S, Joshi K, Jain H, Upadhyay UM. Aquasomes- A self-assembled nanotechnology system. Int J Res Pharm Sci 2011;2(3):492-496.

30. Patel S, Aundhia C, Seth A, Shah N, Pandya K, Patel D. Aquasomes: A novel approach in drug carrier system. Eur J Pharm Med Res2016;3(9):198-201.
31. Kutlehria A, Kaushik P, Sharma S, Kaur A. Aquasomes as a carrier system for oral delivery of Bromelain. Int Res J Pharm 2018;9(8):123-9.

32. Patel JK, Patel KN, Patel HK, Patel BA, Patel PA. Aquasomes: A self-assembling nanobiopharmaceutical carrier system forbioactive molecules: A review. Int J Pharm Res 2012;1(1):11-21.

33. Banerjee S, Sen KK. Aquasomes: A novel nanoparticulate drug carrier. J Drug Deliv Sci Technol 2018;43:446-452.

34. Jain SS, Jagtap PS, Dand NM, Jadhav KR, Kadam VJ. Aquasomes: a novel drug carrier. J Appl Pharm 2012;2(1):184-192.

35. Oviedo IR, Lopez RA, Gasga JR, Barreda CT. Elaboration and structural analysis of aquasomes loaded with indomethacin. Eur J Pharm Sci 2007;32:223-230.

36. Nielsen SS. Phenol-sulphuric acid method for total carbohydrates. Food Science Texts Series 2009:47-53.

37. Vengala P, Subrahmanyam CVS, Gangaraju M.In vitro and in vivo evaluationof Piroxicam loaded ceramic Nanoparticles. Int J Pharma Sci Res 2016;7(7):303-309.

38. Damera DP, Kaja S, Janardhanam LS, Alim SK, Venuganti VV, Nag A. Synthesis, detailed characterization, and dual drug delivery application of BSA Loaded Aquasomes. Appl Bio Mater 2019;2:4471-4484.

39. Chavda V. Aquasomes: Anattractive niche as peptide and protein carrier. Pharm Tech Medica 2013;2(6): 387-395

40. Gualati M, Singh S, Kishore U, Sachdeva RK, Umashankar MS. Potential applications of aquasomes fortherapeutic delivery of proteins andpeptides. Nanostr Drug Deliv 2015;4:439-453.

41. Asfour MH. Advanced trends in protein and peptide drug delivery: a specialemphasis on aquasomes and micro needles techniques. Drug Deliv Transl Res 2020:1-23.

42. Kossovsky N, Gelman A, Rajguru S, Nguyen R, Sponsler E, Hnatyszyn H, et al. Control of molecular polymorphisms by a structured carbohydrate/ceramic delivery vehicle - aquasomes. J Cont Rel 1996;39:383-388.

43. Prasanthi NL, Manikiran SS, Krishna SC, Rao NR. Aquasomes: role to deliver bioactive substances. Res J Pharma Dosage Forms Tech 2010;2(6):356-360.

44. Chaudhary JS, PrjapatiSK, Gupta R. Aquasomes; a new approach for delivering therapeutics: An Overview. Asian J Pharm 2018;12(2): S419.

45. Rawat M, Singh D, Saraf S, Saraf S. Development andin vitro evaluation of alginate gel-encapsulated,chitosan-coated ceramic nanocores for oral delivery ofenzyme. Drug Dev Ind Pharm 2008;34(2):181-188.

46. Tiwari T, Khan S, Rao, N, Josh, A, Dubey, BK. Preparation and characterization ofaquasome based formulations of dithranolfor the treatment of Psoriasis. World J Pharm Sci 2012;1:250-272.

47. Nanjwade BK, Hiremath GM, Manvi FV, Srichana T. Formulation and evaluation ofetoposide loaded aquasomes. J Nano Pharm Drug Deliv 2013;1:92-101.

48. Mizushima Y, Ikoma T, Tanaka J, Hoshi K, Ishihara T, Ogawa Y. Injectable poroushydroxyapatite microparticles as a new carrier for protein and lipophilic drugs. J Contr Rel 2006;110:260-265.

49. Vyas SP, Goyal AK, Khatri K, Mishra N, Mehta A, Vaidya B. Aquasomes: A Nanoparticulate approach for the delivery of antigen. Drug Dev Ind Pharm. 2008;34:1297-1305.

50. Mitragotri S, Burke P, Langer R. Overcoming the challenges in administering biopharmaceuticals: formulation and delivery strategies. Nat Rev Drug Disc 2014;13:655-672. 